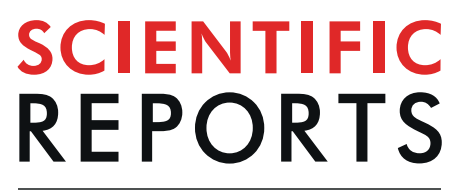

natureresearch

\title{
Genomic Patterns of Homozygosity in Chinese Local Cattle
}

\author{
Lingyang X $\mathrm{u}^{1,3^{*}}$, Guoyao Zhao ${ }^{1,3}$, Liu Yang ${ }^{1}$, Bo Zhu ${ }^{1}$, Yan Chen ${ }^{1}{ }^{1}$, Lupei Zhang ${ }^{1}$, Xue Gao ${ }^{1}$, \\ Huijiang Gao ${ }^{1}$, George E. Liu $\mathbb{D}^{2}$ \& Junya $\mathrm{Li}^{1^{*}}$
}

Genome-wide single nucleotide polymorphism (SNP) arrays can be used to explore homozygosity segments, where two haplotypes inherited from the parents are identical. In this study, we identified a total of 27,358 runs of homozygosity $(\mathrm{ROH})$ with an average of $153 \mathrm{ROH}$ events per animal in Chinese local cattle. The sizes of ROH events varied considerably ranging from 0.5 to $66 \mathrm{Mb}$, with an average length of $1.22 \mathrm{Mb}$. The highest average proportion of the genome covered by ROH ( $11.54 \%$ of the cattle genome) was found in Nanda cattle (NDC) from South China, whereas the lowest average proportion ( $\sim 3.1 \%)$ was observed in Yanhuang cattle $(\mathrm{YHC})$. The average estimated $\mathrm{F}_{\mathrm{ROH}}$ ranged from 0.03 in YHC to 0.12 in NDC. For each of three ROH classes with different sizes (Small 0.5-1 Mb, Medium 1-5 $\mathrm{Mb}$ and Large $>5 \mathrm{Mb}$ ), the numbers and total lengths of $\mathrm{ROH}$ per individual showed considerable differences across breeds. Moreover, we obtained 993 to $3603 \mathrm{ROH}$ hotspots (which were defined where ROH frequency at a SNP within each breed exceeded the $1 \%$ threshold) among eight cattle breeds. Our results also revealed several candidate genes embedded with ROH hotspots which may be related to environmental conditions and local adaptation. In conclusion, we generated baselines for homozygosity patterns in diverse Chinese cattle breeds. Our results suggested that selection has, at least partially, played a role with other factors in shaping the genomic patterns of ROH in Chinese local cattle and might provide valuable insights for understanding the genetic basis of economic and adaptive traits.

Runs of homozygosity $(\mathrm{ROH})$ are defined by contiguous homozygous segments where the two haplotypes transmitted from the parents are identical ${ }^{1}$. The occurrence of $\mathrm{ROH}$ within a population can be influenced by both reduced effective population size in the past and recent inbreeding. In addition, potential effect from genetic drift may also contribute to the formation of ROH. The availability of high-density SNP arrays has promoted the recent studies of regions of autozygosity. Initially, $\mathrm{ROH}$ analysis was utilized as a strategy to map recessive diseases genes in human ${ }^{2,3}$. Genome-wide analysis of homozygosity and their variation across individuals can offer important insights into genetic diversity and demographic history ${ }^{4}$. Abundance of $\mathrm{ROH}$ and their directional dominance on complex traits have been demonstrated in diverse human population ${ }^{5}$. Previous studies have investigated both genomic and geographic distribution of $\mathrm{ROH}$ and their contribution to population history. In human, recent studies suggested the total number and length of $\mathrm{ROH}$ per individual display considerable variation in worldwide human populations ${ }^{6}$, and the genomic distribution of ROH followed an obvious South to North gradient which was in consistent with European population history ${ }^{7}$.

$\mathrm{ROH}$ has also been widely utilized in farm animals to characterize population structure and demography history. Also, analyses of $\mathrm{ROH}$ can help to disclose the genetic relationships among individuals, measure the genome inbreeding level, and identify the region associated with economically important trait ${ }^{8-20}$. In cattle, Purfield et al. assessed the level of $\mathrm{ROH}$ in a wide range of daily and beef cattle breeds ${ }^{11}$, and their findings suggested different distribution patterns of $\mathrm{ROH}$ length may imply variations in breed origins and recent management among them. Extensive studies have been performed for evaluation of inbreeding and their negative effects on production and reproductive ability based on $\mathrm{ROH}$ in commercial daily cattle ${ }^{15,16,18}$. Also, several studies have been conducted to estimate inbreeding depression and investigate climatic adaptation in local cattle ${ }^{14,21}$.

Previous studies suggested the occurrence of $\mathrm{ROH}$ is not randomly distributed across the genomes, and $\mathrm{ROH}$ hotspots appear to be shared among individuals, which is probably caused by selection pressure ${ }^{22}$. The autozygosity across genome can help to improve the understanding of selection process and identify potential candidate

${ }^{1}$ Innovation Team of Cattle Genetic Breeding, Institute of Animal Sciences, Chinese Academy of Agricultural Sciences, Beijing, 100193, China. ${ }^{2}$ Animal Genomics and Improvement Laboratory, Department of Agriculture-Agricultural Research Services, Beltsville, Maryland, 20705, United States of America. ${ }^{3}$ These authors contributed equally: Lingyang Xu and Guoyao Zhao. *email: xulingyang@163.com; lijunya@caas.cn 


\begin{tabular}{|c|c|c|c|c|c|c|c|c|}
\hline & $\begin{array}{l}\text { Sample } \\
\text { Size }\end{array}$ & \begin{tabular}{|l|l} 
Total \\
Number $^{\mathrm{a}}$
\end{tabular} & $\begin{array}{l}\text { Average Number } \\
\text { per ind }\end{array}$ & $\begin{array}{l}\text { Total length } \\
(\mathrm{Mb})^{\mathbf{b}}\end{array}$ & $\begin{array}{l}\text { Average length } \\
\text { Per ind }(\mathrm{Mb})\end{array}$ & $\begin{array}{l}\text { ROH-region } \\
{\text { length }(\mathrm{Mb})^{\mathrm{c}}}\end{array}$ & $\begin{array}{l}\text { ROH-region } \\
\text { number }^{d}\end{array}$ & $\begin{array}{l}\text { Genome } \\
\text { Coverage }^{\mathrm{e}}\end{array}$ \\
\hline YHC & 21 & \begin{tabular}{|l}
1889 \\
\end{tabular} & 90 & 1821 & 87 & 1135 & 759 & \begin{tabular}{|l|}
0.41 \\
\end{tabular} \\
\hline MGC & 21 & 2156 & 103 & 4859 & 231 & 1906 & 423 & \begin{tabular}{|l|l|}
0.68 \\
\end{tabular} \\
\hline CDM & 25 & 2167 & 87 & 4900 & 196 & 2078 & 329 & \begin{tabular}{|l|l|}
0.74 \\
\end{tabular} \\
\hline PWC & 23 & 2601 & 113 & 3389 & 147 & 1675 & 721 & \begin{tabular}{|l|l|}
0.60 \\
\end{tabular} \\
\hline LSC & 22 & 2873 & 131 & 2397 & 109 & 1297 & 1011 & \begin{tabular}{|l|l|}
0.46 \\
\end{tabular} \\
\hline ZTC & 23 & 2861 & 124 & 3496 & 152 & 1800 & 650 & \begin{tabular}{|l|l|}
0.64 \\
\end{tabular} \\
\hline WSC & 21 & 4741 & 226 & 4935 & 235 & 1957 & 755 & \begin{tabular}{|l|l|}
0.70 \\
\end{tabular} \\
\hline NDC & 23 & 8070 & 351 & 7431 & 323 & 2129 & 770 & \begin{tabular}{|l|l|}
0.76 \\
\end{tabular} \\
\hline
\end{tabular}

Table 1. The descriptive statistics of $\mathrm{ROH}$ and $\mathrm{ROH}$ region for eight Chinese local cattle breeds. Note: ${ }^{\text {aThe }}$ total number of $\mathrm{ROH}$ events for each breed. ${ }^{\mathrm{b}}$ The total length of $\mathrm{ROH}$ events across individuals for each breed. ${ }^{\mathrm{c}}$ The length of $\mathrm{ROH}$ region for each breed. ${ }^{\mathrm{d}}$ The number of nonredundant $\mathrm{ROH}$ region for each breed. ${ }^{\mathrm{e}} \mathrm{The}$ proportion of $\mathrm{ROH}$ coverage of genome for each breed.

genes associated with important traits in cattle ${ }^{23-29}$. The formation of $\mathrm{ROH}$ of various sizes (long vs. short) may reflect different evolutionary events on recent vs. ancient inbreeding ${ }^{6,12}$, with long ROH indicate more recent inbreeding, while short $\mathrm{ROH}$ suggest more ancient inbreeding. The investigation of these $\mathrm{ROH}$ segments can further improve our understanding of selection histories for special traits among diverse populations ${ }^{23,24}$.

Most of Chinese local cattle have been isolated in local feed environments, thus the potential homozygosity regions could be generated among them, which may also be influenced by adaptive selection and other factors in specific environment ${ }^{30-32}$. Despite several studies have conducted on analysis of population structures and admixture in Chinese local cattle ${ }^{31,33}$, no comprehensive study has been reported to explore the ROH pattern using high density SNP arrays. The aims of the present study were to (i) investigate genome-wide autozygosity patterns and genomic inbreeding level in Chinese local cattle; (ii) characterize profiles of ROH with different sizes and their gene contents with ROH hotspots; (iii) evaluate potential diverse selection based on the landscape of $\mathrm{ROH}$ across genome.

\section{Results}

Genomic ROH distribution. We identified a total of 27,358 ROH with an average of 153 per individual. The ROH size varied considerably from 0.5 to $66 \mathrm{Mb}$, with an average size of $1.22 \mathrm{Mb}$ across all autosomes (Supplementary File 1: Table S1). The descriptive statistics of $\mathrm{ROH}$ and nonredundant $\mathrm{ROH}$ region per breed were presented in Table 1. Based on geographical locations, 8 breeds can be divided into 4 groups including North group (YHC and MGC), Northwest group (CDM), Southwest group (PWC, LSC and ZTC) and South group (WSC and NDC). We found cattle breeds from South and Southwest groups had the higher average ROH numbers, while cattle from North group had lower average numbers. The number of ROH per animal varied from 87 in CDM to 351 in NDC cattle (Table 1). Also, we observed the highest proportion of the genome covered by $\mathrm{ROH}$ in NDC (average ROHlength per individual was $323 \mathrm{Mb}$, corresponding to $\sim 11.54 \%$ of cattle genome) from South China, whereas the lowest was found in YHC (average ROHlength per individual was $87 \mathrm{Mb}$, corresponding to $3.1 \%$ ). As expected, the proportion of the genome coverage by $\mathrm{ROH}$ for each breed showed high correlation with their average observed heterozygosity $(\mathrm{Ho})$ with $r=0.71$. In addition, we found the numbers and sizes of $\mathrm{ROH}$ events per individual varied across populations. NDC had larger average numbers and sizes of ROH events than other breeds (Fig. 1).

ROH region and inbreeding coefficients. $\mathrm{ROH}$ regions were determined by merging $\mathrm{ROH}$ identified across all individuals using previously published protocols implemented in BEDTools ${ }^{34}$. Totally, we identified 86 nonredundant $\mathrm{ROH}$ regions in Chinese local cattle, covering $2.47 \mathrm{~Gb}$ of the cattle genome (Supplementary File 2: Table S2). We observed the total length of merged $\mathrm{ROH}$ regions ranged from $1.13 \mathrm{~Gb}$ ( $40.53 \%$ of genome) in YHC to $2.12 \mathrm{~Gb}(\sim 76.02 \%$ of genome) in NDC (Table 1$)$. Next, we estimated inbreeding coefficients within each population using three methods including $\mathrm{F}_{\mathrm{ROH}}, \mathrm{F}_{\mathrm{HOM}}$ and $\mathrm{F}_{\mathrm{GRM}}$. The individual $\mathrm{F}_{\mathrm{ROH}}$ values varied from 0.01 to 0.34 , while the highest average $\mathrm{F}_{\mathrm{ROH}}(0.12)$ was estimated in $\mathrm{NDC}$. The $\mathrm{F}_{\mathrm{GRM}}$ values were lower when compared to $\mathrm{F}_{\mathrm{ROH}}$, which ranged from -0.07 to 0.36 , whereas the coefficient for $\mathrm{F}_{\mathrm{HOM}}$ ranged from -0.15 to 0.33 . The highest

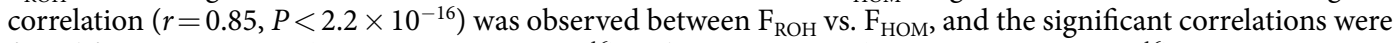
found for $\mathrm{F}_{\mathrm{ROH}}$ vs. $\mathrm{F}_{\mathrm{GRM}}\left(\mathrm{r}=0.78, P<2.2 \times 10^{-16}\right)$ and $\mathrm{F}_{\mathrm{HOM}}$ vs. $\mathrm{F}_{\mathrm{GRM}}\left(r=0.81, P<2.2 \times 10^{-16}\right)$.

Genomic patterns of homozygosity. To investigate the $\mathrm{ROH}$ pattern, we divided them into three size classes: A. Small ( $500 \mathrm{~kb}$ to $1 \mathrm{Mb}$ ), B. Medium (1 Mb to $5 \mathrm{Mb}$ ), and C. Large ( $>5 \mathrm{Mb}$ ), as described in the previous study $^{12}$. The ROH distributions by total length and number for each group were shown in Fig. 2 . The proportion of the genome in $\mathrm{ROH}$ of different lengths (thus inferred to be autozygous) varied clearly among breeds.

Our result showed two breeds (MGC and CDM) had the largest total lengths of Large ROH ( $>5 \mathrm{Mb}$, Fig. 2A) as compared to the other breeds, while the LSC had the smallest total length. Moreover, Small and Medium ROH were found to be predominant in the South group breeds (NDC and WSC) by their total lengths. Our results also revealed that Small ROH predominated across Southwest groups of Chinese local cattle (Fig. 2B). The total numbers of Small and Median ROH showed increasing trends for cattle from North to South groups. 

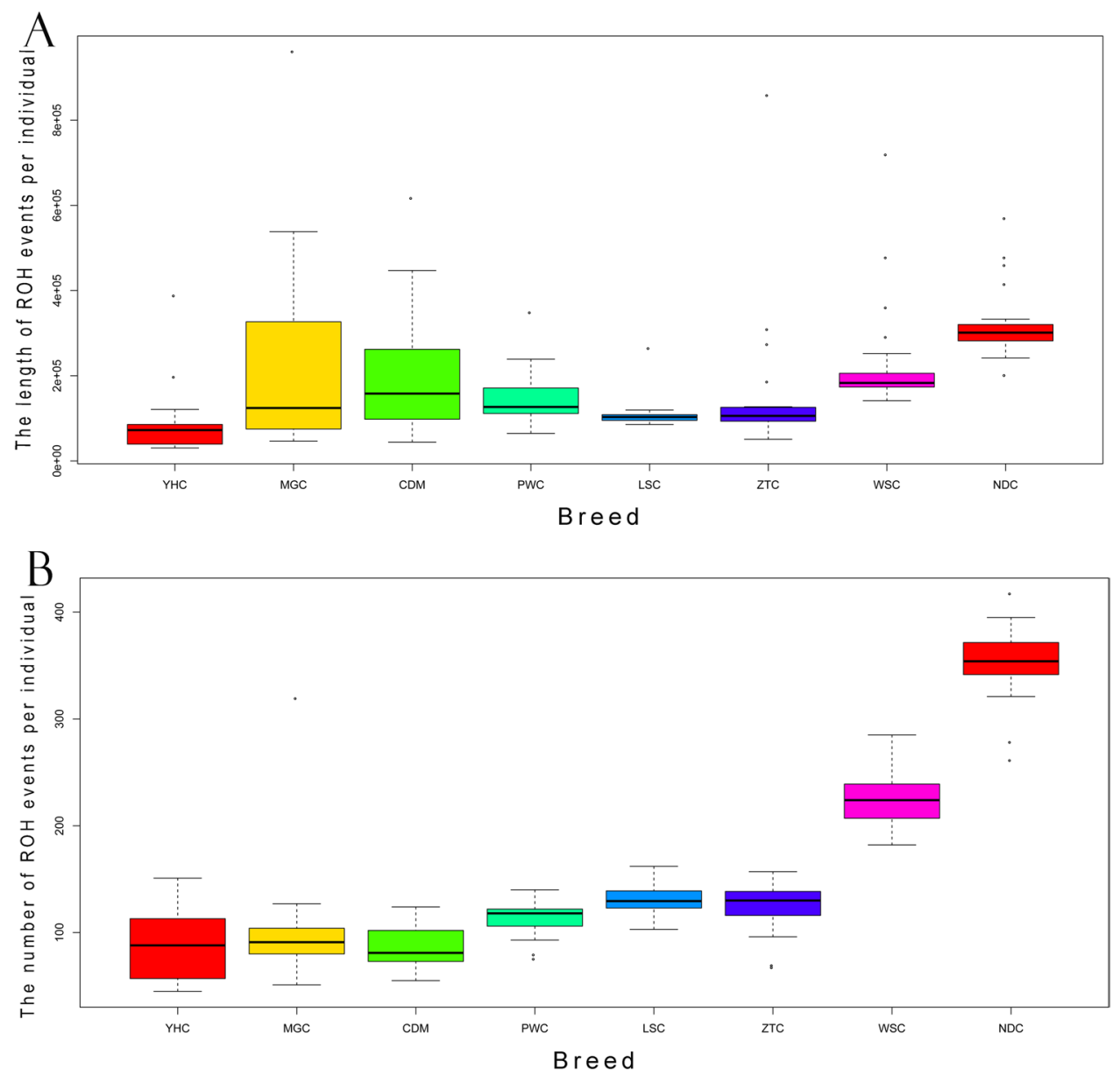

Figure 1. Individual patterns of $\mathrm{ROH}$. The distributions of $\mathrm{ROH}$ statistics per individual for eight Chinese local cattle breeds, including YHC $(n=21)$, MGC $(n=21), \operatorname{CDM}(n=25)$, PWC $(n=23)$, LSC $(n=22)$, ZTC $(n=23)$, WSC $(n=21)$, and NDC $(n=23)$. Groups are divided based on geographic locations in China (North, Northwest, South and Southwest). (A) The length of $\mathrm{ROH}$ events per individual. (B) The number of ROH events per individual.

As previously reported, the likelihood of $\mathrm{ROH}$ occurrence at a particular chromosomal position generally depends on the length of $\mathrm{ROH}^{12}$. We found that the distributions of $\mathrm{ROH}$ occurrence along the autosomes vary among eight breeds. The high proportion of $\mathrm{ROH}$ mostly appeared in the middle of the chromosomes with the low recombination rates, and much less towards the telomeric regions (Fig. 3). Additionally, NDC and WSC have high proportion of $\mathrm{ROH}$ across chromosomes when compared to other breeds.

ROH profiles across populations. To study the demographic history, we firstly plotted the numbers of $\mathrm{ROH}$ per individual against the total length of them (Fig. 4A). We observed ROH profiles (length vs. number) displayed different patterns among eight breeds. Out of 8 cattle breeds, YHC showed a small number of ROH and short total length in $\mathrm{ROH}$ with most points locating in the left corner of the plot (Fig. 4A). MGC, CDM, ZTC, and PWC had most points in the higher left corner of the plot, indicating a small number of $\mathrm{ROH}$, but a large sum of total ROH length. WSC and NDC were extreme cases with large numbers of Small and Medium ROH and large total $\mathrm{ROH}$ lengths. Also, we observed the different patterns of $\mathrm{ROH}$ when $\mathrm{ROH}$ were classified into three class by sizes, including Large (Fig. 4B), Medium (Fig. 4C) and Small classes (Fig. 4D). Our results suggested Medium and Large $\mathrm{ROH}$ showed large differences among individuals.

To assess the genomic distribution patterns of total $\mathrm{ROH}$ length, we also conducted pairwise comparisons among the total lengths of three classes of Small, Medium and Large ROH in individual cattle genomes (Fig. 5). We found the total lengths of Small and Medium were highly correlated $\left(r=0.72, P<2.2 \times 10^{-16}\right)$, while none of them is strongly correlated with the total length of Large $\left(r=0.36\right.$ with $P<5.36 \times 10^{-7}$ and $r=-0.19$ with $\mathrm{p}<0.0106$, respectively).

ROH involved with selection signatures. To investigate effect of selection in diverse cattle, we explored the occurrences of $\mathrm{ROH}$ across genome. The frequency of a SNP locus (\%) in those ROH was assessed for each breed, and then plotted against the position of the SNP along autosomes (Fig. 6). The threshold for each breed were $0.29,0.48,0.4,0.30,0.32,0.30,0.57,0.74$ for YHC, MGC, CDM, PWC, LSC, ZTC, WSC and NDC cattle. Our results suggested that the occurrences of $\mathrm{ROH}$ varied clearly within the genome among diverse cattle. We 

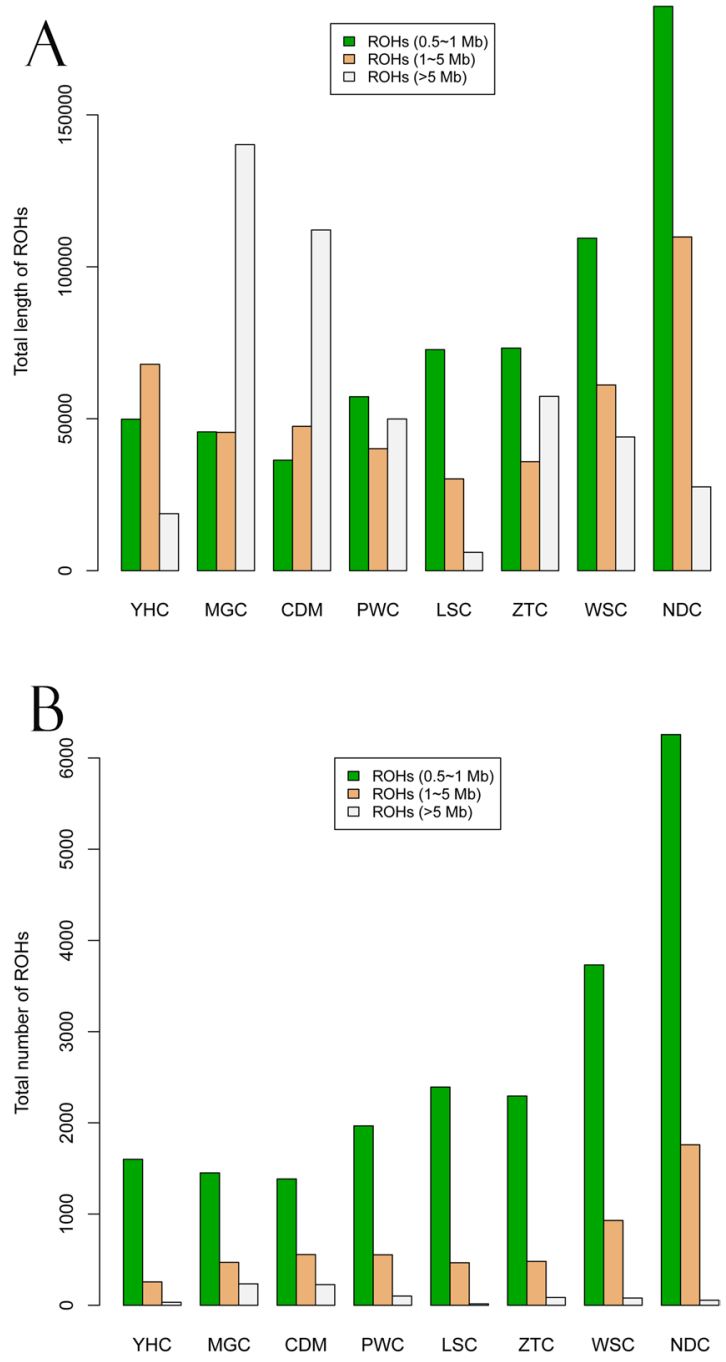

Figure 2. Total length and number of $\mathrm{ROH}$ in Chinese local cattle. (A) The total length of $\mathrm{ROH}$ belonging to three size classes including Small $(0.5$ to $1 \mathrm{Mb})$, Medium $(1$ to $5 \mathrm{Mb})$ and Large ( $>5 \mathrm{Mb})$ size classes for each of the different populations. (B) The total number of ROH belonging to three size classes.

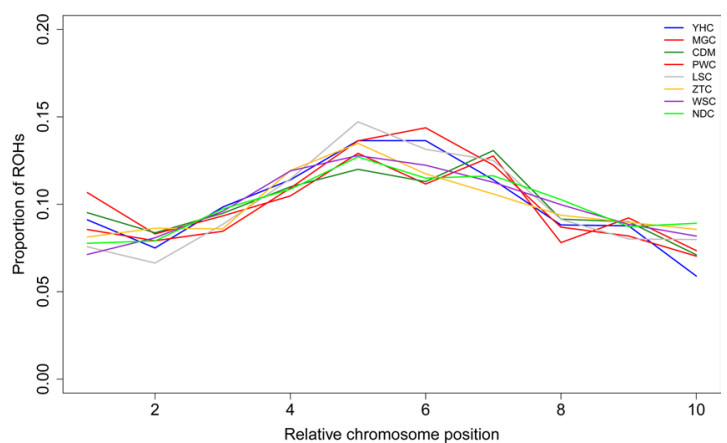

Figure 3. ROH distribution over relative chromosomal position in Chinese local cattle, and the distributions of ROH bins are averaged over all chromosomes.

observed 3603, 1542, 1055, 2195, 3216, 1839, 1444 and 993 hotspots at top 1\% in YHC, MGC, CDM, PWC, LSC, ZTC, WSC and NDC cattle, respectively. Within the genomic regions with a high level of autozygosity under potential selection, we identified 382 unique genes across eight breeds. Among them, twelve genes including CTNNA1, LRRTM2, SIL1, SNHG4, MATR3, PAIP2, MZB1, SPATA24, DNAJC18, ECSCR, TMEM173, UBE2D2 were shared across eight breeds. The DAVID annotations for these top annotated genes for each of eight breeds were presented in Supplementary File 3: Table S3. Most genes identified in North and Norwest group, including 
A
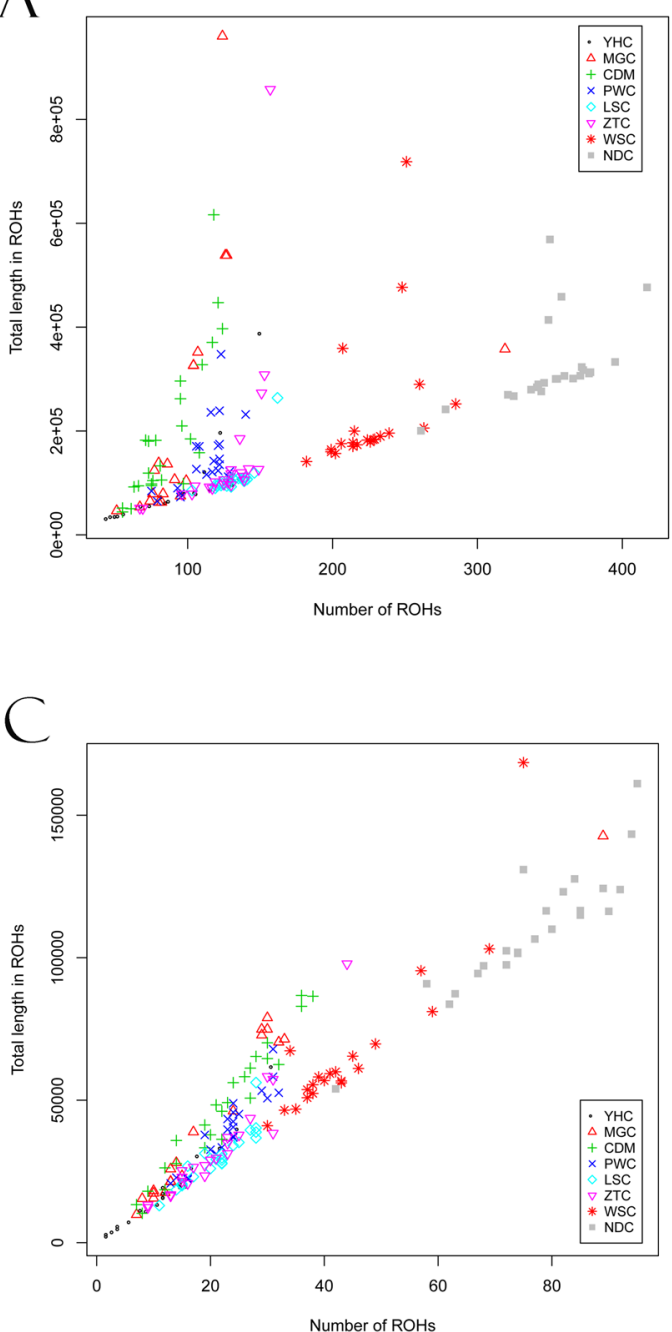

B
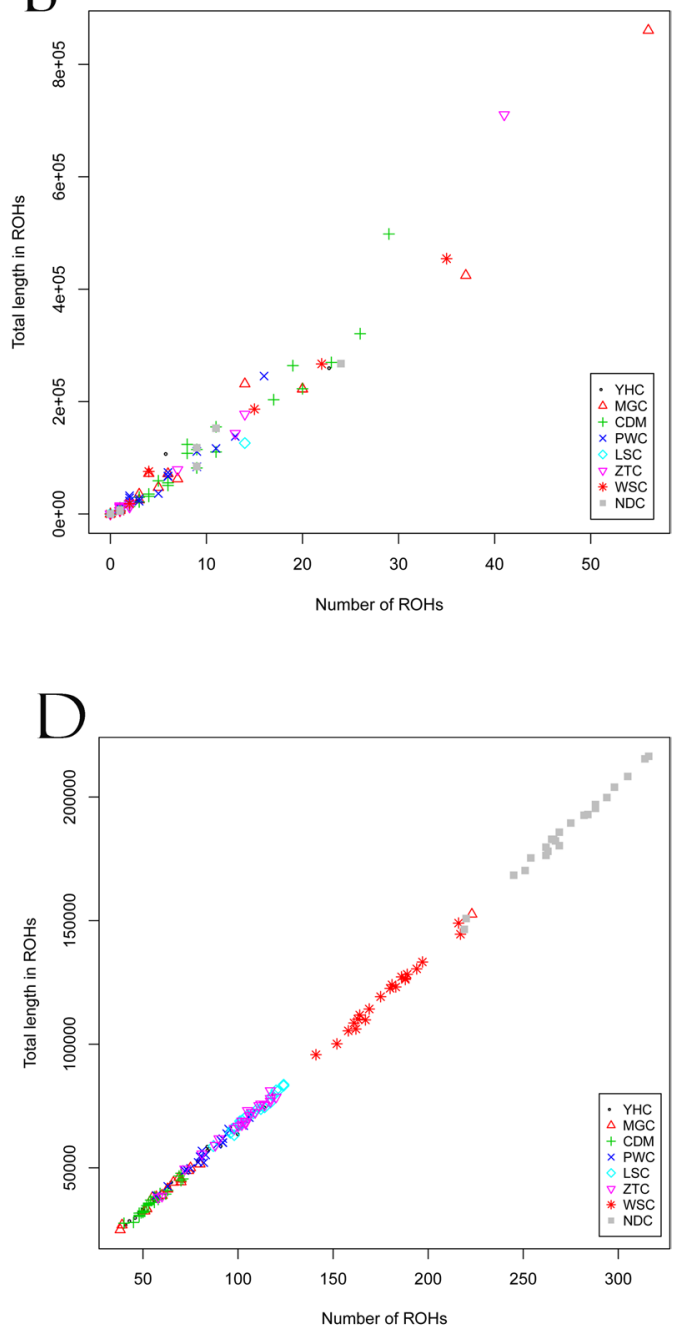

Figure 4. Evaluation of number of $\mathrm{ROH}$ and $\mathrm{ROH}$ total length for eight cattle populations. The number of $\mathrm{ROH}$ found for each individual genome $(x$-axis) is plotted against $\mathrm{ROH}$ total length (i.e. the length of $\mathrm{Mb}$ covered by ROH in each genome, $y$-axis). We analyzed cattle from YHC, MGC, CDM, LSC, PWC, ZTC, WSC and NDC. (A) All ROH; (B) size class Large (>5Mb); (C) size class Medium (1 to $5 \mathrm{Mb}$ ) and (D) size class Small (0.5 to $1 \mathrm{Mb})$.

MGC and CDM, were enriched in lysozyme activity, cytolysis, defense response to Gram-negative bacterium and cell wall macromolecule catabolic process.

Among 382 unique genes, a total of 137 were identified in two and more populations. In YHC, two regions were detected on BTA7 and BTA21, including two genes (ETF1 and HSPA9) at $51.5 \mathrm{Mb}$ on BTA7 and three genes (TMEM266, ETFA and ISL2) at $32 \mathrm{Mb}$ on BTA21. In MGC, we detected top three regions on BTA7, BTA11 and BTA23: the top region on BTA7 overlapped with six genes CTNNA1, LRRTM2, SIL1, SNHG4, MATR3 and PAIP2, while no known gene was found within the regions on BTA11 and BTA23. Also, we observed the top three regions on BTA5, BTA13 and BTA14 in CDM. Among them, the top region on BTA5 contained genes YEATS4 and LYZ1. We found 17 genes were included in the region ranging from 54.48 to $54.85 \mathrm{Mb}$ on BTA13, while no known gene was found within the region on BTA14. In the LSC cattle, we observed one significant region on each of BTA5, BTA7 and BTA16, which overlapped with many genes and spanned $0.6 \mathrm{Mb}, 7 \mathrm{Mb}$ and $0.1 \mathrm{Mb}$, respectively. While in the ZTC, only one region surpassed the $50 \%$ occurrence of $\mathrm{ROH}$ which ranged from 51.6 to $52.7 \mathrm{Mb}$, containing the important gene like CTNNA1, ISL2, DNAJC18, and SPATA24. Finally, no significant region ( $>50 \%$ occurrence) was detected for the PWC in Southwest groups. For two breeds in South group, we observed that multiple regions on BTA2, BTA5, BTA7, BTA11, BTA16, BTA14 and BTA22 were shared by WSC and NDC within the regions of above $50 \%$ occurrences.

In addition, a total of 247 genes were identified in unique breed based on the occurrence of $\mathrm{ROH}$, of those, $56,9,15,69,28,38,24,8$ genes were uniquely identified in YHC, MGC, CDM, LSC, PWC, ZTC, WSC and NDC, respectively. 

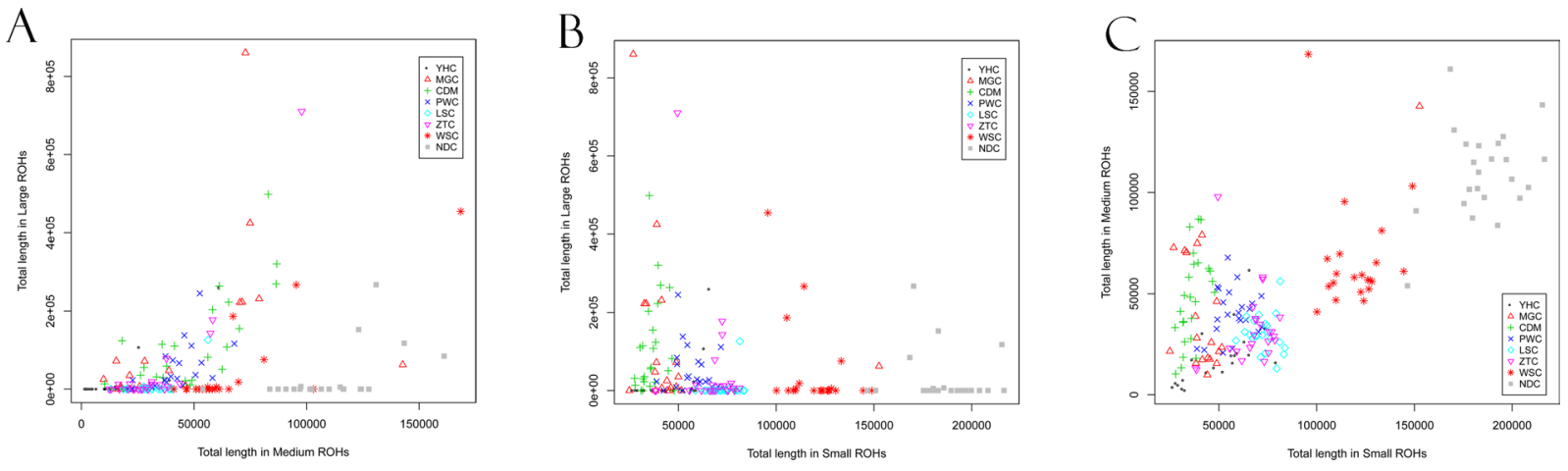

Figure 5. Comparison of total lengths of $\mathrm{ROH}$ per individual across size classes, including Small, Medium and Large. Pairwise correlations and their significance levels between the total lengths of Small, Medium, and Large $\mathrm{ROH}$ in individual genomes were estimated using Pearson correlations. (A) Large versus Medium $(\mathrm{r}=0.72)$. (B) Large versus Small $(\mathrm{r}=0.36)$. (C) Medium versus Small $(\mathrm{r}=-0.19)$.

A

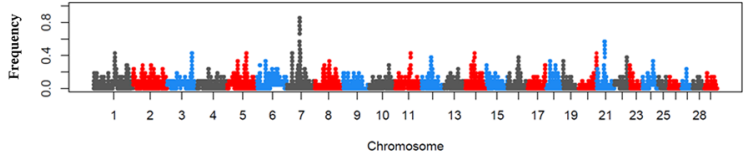

B
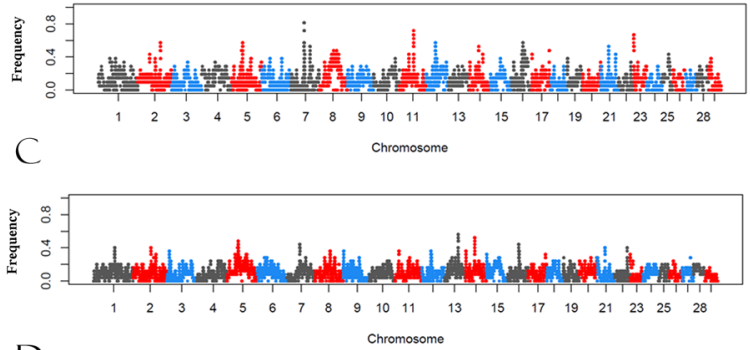

$\mathrm{D}$

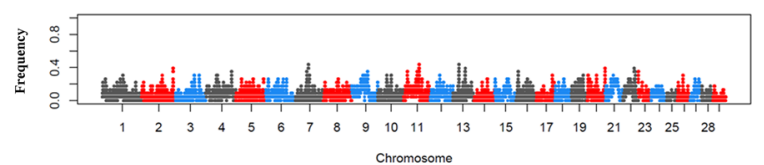

E

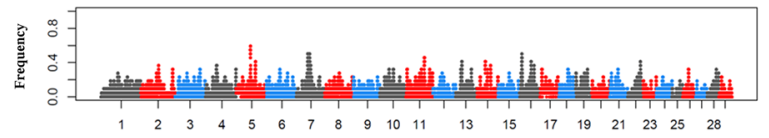

F

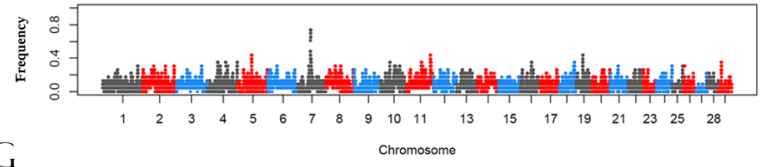

G

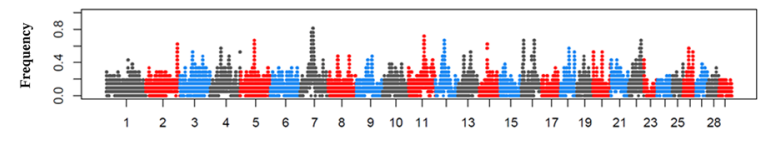

$\mathrm{H}$

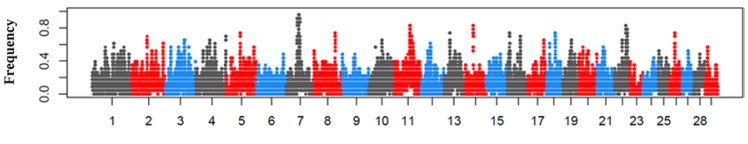

Figure 6. Manhattan plot of the distribution of runs of homozygosity (ROH) hotspots in the Chinese local cattle genome. The $x$-axis represents the SNP genomic coordinate, and the $y$-axis shows the frequency (\%) of overlapping ROH shared among individuals. (A) YHC; (B) MGC; (C) CDM; (D) PWC; (E) LSC; (F) ZTC; (G) WSC; and (H) NDC.

\section{Discussion}

Cattle represent important genetic resources that contribute to local economy. Many studies have been explored the $\mathrm{ROH}$ pattern and its associated inbreeding depression related to traits at the genomic level in multiple cattle population ${ }^{8}$. However, the $\mathrm{ROH}$ patterns and their distributions in Chinese local cattle remain largely unexplored. To our knowledge, this study is the first attempt to investigate the occurrence and distribution of $\mathrm{ROH}$ in Chinese local cattle using high-density SNP arrays. The high density SNP arrays are more sensitive for the determination of small segments, as previously reported ${ }^{35}$, while the BovineSNP50K array may underestimate the numbers of segments of 1 to $4 \mathrm{Mb}$ in length.

We estimated inbreeding coefficients within each population using three methods including $\mathrm{F}_{\mathrm{ROH}}, \mathrm{F}_{\mathrm{HOM}}$ and $\mathrm{F}_{\mathrm{GRM}}$. In general, the correlations among $\mathrm{F}_{\mathrm{ROH}}, \mathrm{F}_{\mathrm{HOM}}$ and $\mathrm{F}_{\mathrm{GRM}}$ varied dramatically across studies. Our study revealed the relative high correlation $(0.78 \sim 0.85)$ among them, and high correlations between $\mathrm{F}_{\mathrm{ROH}}$ and the two other estimates of genomic inbreeding $\left(\mathrm{F}_{\mathrm{GRM}}\right.$ and $\left.\mathrm{F}_{\mathrm{HOM}}\right)$ can be considered as an accurate estimator of the IBD genomic proportion ${ }^{17}$. On the other hand, several other studies have found a low to moderate $\mathrm{F}_{\mathrm{GRM}}-\mathrm{F}_{\mathrm{ROH}}$ correlation for dairy breeds ${ }^{36,37}$, while moderate to high correlations were reported in Holstein cattle and Jersey populations ${ }^{15,38}$. We suspected that these different results could be related to ROH type, sample populations and SNP array platforms.

In this study, we found clear differences in terms of total number and length of ROH in diverse Chinese local cattle, and the relationship between them varied considerably among individuals. This was consistent with 
previous studies with large-scale datasets in cattle, their findings suggested that $\mathrm{ROH}$ are often differentially distributed across breeds, and the patterns of $\mathrm{ROH}$ (number and length) may imply differences in breed origins and recent management ${ }^{11}$. The British Isles breeds including Hereford, Guernsey, Angus and Jersey cattle displayed the highest sum of $\mathrm{ROH}$ per animal compared other breeds, while African breeds displayed high variability in total number of ROH among breeds ${ }^{11}$.

A recent study revealed that the average total length of $\mathrm{ROH}$ ranged from $106 \mathrm{Mb}$ in Piedmontese to $371 \mathrm{Mb}$ in Brown cattle ${ }^{36}$, and their study suggested that dairy breeds have longer and larger size $\mathrm{ROH}$ than beef and dual-purpose cattle, and inbreeding is more recent in these dairy breeds. In our study, we found some breeds like WSC and NDC in South China displayed larger ROH size, which may reflect the effects of current inbreeding. We suspected that both breeds may be selected in isolated conditions with relative small effective population sizes. This finding also implied that continuing isolation and a reduced population size might play an essential role in the formation of $\mathrm{ROH}$.

The distribution of $\mathrm{ROH}$ numbers and lengths can illustrate the genetic diversity of studied population. We also observed diverse pattern between the total number and length of $\mathrm{ROH}$ in Chinese local cattle, which were agree with the previous goat and sheep studies ${ }^{20,28}$. A large proportion of individuals with large number and length were identified in NDC and WSC (Fig. 4A). In contrast, for the Small ROHs in each individual, the total length of Small ROH in NDC and WSC mostly ranged from 100 to $250 \mathrm{Mb}$ and none of them was less than $100 \mathrm{Mb}$ (Fig. 4D). This finding reflected that both ancient and recent inbreeding had a critical impact on cattle genomes. This $\mathrm{ROH}$ pattern might be caused by the breeding isolation of these populations. Additionally, the NDC and WSC have relatively small populations and might have experienced bottlenecks as indicated by high fraction of the genome in ROH. On the other hand, YHC showed very low amounts of ROH (Fig. 4A). This is consistent with recent admixture in the individuals from YHC. Otherwise, we observed medium level ROH in terms of both total length and number of ROH in CDM and PWC. This probably reflects an absence of admixture in those breeds. The comparison for distribution of $\mathrm{ROH}$ numbers and lengths may be useful to demonstrate the genetic diversity among cattle breeds.

A recent study assessed the pattern of homozygosity and they found the average coverage of genome with autozygosity was $175 \mathrm{Mb}$ in Gyr (Bos indicus) cattle ${ }^{17}$, while our study found the average value of ROH coverage per animal were 323, 235 and $231 \mathrm{Mb}$ in NDC, WSC and MGC, respectively. We also observed the cumulative ROH length was dominated by an abundance of Small to Medium ROH in WSC as well as NDC, which implied either of them is an isolated breed based on a source population of substantial size. Abundance of ROH due to genetic isolation has also been reported in European cattle populations ${ }^{13}$ and Japanese wild boar ${ }^{12}$. The accumulation of large $\mathrm{ROH}$ in individuals of MGC and CDM could be caused by potential selection pressure for special environmental conditions of these populations, which has also been reported in Spanish local goat breeds ${ }^{20}$. Three $\mathrm{ROH}$ class (Large, Small and Medium) may be influenced via different processes, including recent inbreeding and recombination on different evolutionary scales ${ }^{6}$. Clustering $\mathrm{ROH}$ into different size classes make it possible to detect and interpret genomic difference among breeds. In addition, previous study reported that long $\mathrm{ROH}$ are mostly enriched in regions with deleterious mutations, and inbreeding can elevate the occurrence of rare recessive diseases that represent homozygotes for deleterious mutations ${ }^{39}$. Therefore, control the increase of inbreeding can effectively promote the implement conservation programs and maintain genetic diversity in local cattle breeds.

Also, pairwise comparisons between the total lengths of Small, Medium, and Large $\mathrm{ROH}$ in individual cattle genomes suggested the total lengths of Small and Medium ROH are highly correlated $\left(r=0.72, P<2.2 \times 10^{-16}\right)$. Neither class Small or Medium is strongly correlated with the total length of Large ROH $(r=0.36$ with $P<5.36 \times 10^{-7}$ and $r=-0.19$ with $P<0.0106$, respectively). Our study revealed that most of ROH belongs to the Short to Medium ROH categories. Compared with breeds from Southwest and South, our results suggested that two breeds (MGC and CDM) have been high inbred based on high coverage of Large ROH (Fig. 2A), probably due to a relatively small population size and close mating for each of two breeds. Because long homozygosity segments across chromosome can be interrupted by recombination events, Large $\mathrm{ROH}$ are usually generated by recent inbreeding, while Small/Medium $\mathrm{ROH}$ are indicative of more ancient relatedness. For instance, a previous study highlighted $\mathrm{ROH}$ preferentially occur in regions of decreased recombination activity ${ }^{12}$. And another study reported the existence of recombination hotspots across the cattle genome can influence $\mathrm{ROH}^{11}$. Our finding was consistent with these results, and the identified regions with ROH hotspots frequently coincided with those with lower recombination rates. Moreover, we also found the studied populations display different proportions of ROH across the chromosomes (Fig. 3).

The occurrence of $\mathrm{ROH}$ hotspots in genomic regions that harbor candidate genes may be involved in selection pressure in response to environmental condition. Previous study has investigated homozygosity in Polish Red cattle, which are characterized by high fertility and easy calving, good immunity and health, as well as high milk quality ${ }^{29}$. Their finding revealed four genes regulating signaling lymphocytic activation including SLAMF1, CD48, Ly9 and SLAMF3. In our study, we found several genes within ROH hotspots, which have been previously reported under selection in cattle ${ }^{40-45}$. Among them, we identified Heat Shock Protein Family A (Hsp70) Member 9 (HSPA9) in seven Chinese local breed, this gene is immune-related genes, and also shows differentially expressed during bovine muscle development ${ }^{46}$. Previous studies suggested that HSPA9 abundance in muscle had positive correlation with beef tenderness ${ }^{47,48}$. This regulation of HSPA9 on meat tenderness is likely due to its anti-apoptotic effect, which prevents the formation of protein aggregates ${ }^{49}$. Moreover, we observed several genes like CTNNA1, SIL1, PAIP2 in eight breed, and ZBTB46 and GMEB2 in CDM located within/near the ROH hotspots, which are also involved in selection in goat and sheep $\mathrm{p}^{50-52}$.

To assesses the breed-specific differences in $\mathrm{ROH}$ occurrence related to selection, we investigated the genes within or near the $\mathrm{ROH}$ islands. In our study, we identified one gene named EEF1A2 in CDM, which was reported to be related to fatty acid and potentially regulate meat tenderness ${ }^{53-55}$. Several genes SIRT6, CREB3L3 and B3GNT5 were specifically detected in YHC cattle, which are reported related to energy meat quality, homeostasis and glycan 
synthesis $^{56-59}$. Two genes TCF12 and TXNDC9 uniquely detected in LSC which were related to muscle lipid composition and marbling in beef cattle ${ }^{60,61}$. Moreover, we identified breed-specific genes (BMP2, FGF9 and CD14) related to growth traits and immune process in PWC, WSC and ZTC, respectively ${ }^{62-64}$. Using the ROH approach, we detected many genes which show potential effects for important traits as reported in many previous studies. Our finding further supported that pattern of $\mathrm{ROH}$ can be utilized to explore genomic regions and genes under specific selection, and the shared $\mathrm{ROH}$ may potentially contain alleles associated with important traits. Despite many genes were identified in our $\mathrm{ROH}$ analysis, functional validation of them is still warranted in the future.

It was noted that we compared our settings to some other studies which used PLINK under its default settings ${ }^{11,17,21}$, and found out our settings generally agreed with the most of published studies using the Bovine HD SNP array. For example, Purfield et al. tested a minimum run length of 58 SNPs was needed to produce $<5 \%$ randomly generated $\mathrm{ROH}$ and the maximum gap between two consecutive homozygous SNPs in a run was set at a $100 \mathrm{~kb}^{11}$. We found their options used was like ours, which slides a window of 50 SNPs, in one SNP intervals, across the genome estimating homozygosity. Although a previous simulation study has tested out ROH calling programs and their settings and reported the best program (PLINK) and its optimal settings ${ }^{65}$, the ROH calling methods and their settings are still challenging ${ }^{11,35}$, thus these results including ours should be interpreted by cautions. Also, to avoid bias and overestimation for ROH detection using PLINK, more options should be considered carefully according to the genome size and the number of SNPs genotyped.

In summary, our study revealed the occurrence of autozygosity varied largely across breeds in Chinese local cattle as different recent and ancient events. We further speculate that selection has partially contributed to landscape of $\mathrm{ROH}$ and their functions in the Chinese local cattle.

\section{Materials and Methods}

Ethics statement. All animal experiments were approved by the Chinese Academy of Agricultural Sciences (CAAS, Beijing, China). All animal procedures were performed in strict accordance with the guidelines proposed by the China Council on Animal Care and the Ministry of Agriculture of People's Republic of China.

Genotyped samples. The genome-wide SNP data of 179 samples from eight populations including Yanhuang (YHC), Menggu (MGC), Caidamu(CDM), Liangshan (LSC), Pingwu(PWC), Zhaotong (ZTC), Wenshan (WSC) and Nandan (NDC) were genotyped by Illumina BovineHD SNPs array, which was retrieved from our previous study ${ }^{32}$. The full names, associated abbreviation for each breeds and additional information on the locations of the sampling areas was presented in Supplementary File 4: Table S4. To minimize genetic relationships among samples, we removed closely related individuals if the PI-HAT value was greater than 0.25. Only autosomal SNPs passed following filters were used for subsequently analyses, and all selected samples displayed a genotype calling rate of more than $99 \%$. Individuals with more than $5 \%$ of missing SNPs were removed from further analyses. Moreover, the SNPs were filtered with MAF $<0.05$ (a minor allele frequency higher than 0.95) and geno $<0.1$ (only SNPs with a $90 \%$ genotyping rate or higher).

ROH estimation. ROH were detected across autosomes for each individual using PLINK v1.0766. The ROH were defined by a minimum of $0.5 \mathrm{Mb}$ in length to avoid short and common $\mathrm{ROH}$ that occur throughout the genome due to LD. The PLINK software detect homozygous segments by scanning along genotype for each individual. The following six criteria were considered to define a ROH: (i) a sliding window of 50 SNPs across the genome; (ii) the proportion of homozygous overlapping windows was 0.05 ; (iii) the minimum number of consecutive SNPs included in a $\mathrm{ROH}$ was 100; (vi) a required minimum density was set at $50 \mathrm{~kb} / \mathrm{SNP}$; (v) the minimum length of a ROH was set to $500 \mathrm{~kb}$, the maximum gap between consecutive homozygous SNPs was $100 \mathrm{~kb}$; and (vi) a maximum of two SNPs with missing genotypes and up to one heterozygous genotype were allowed in a $\mathrm{ROH}$.

ROH classification and inbreeding coefficient. ROH were divided into three classes based on length: $0.5-1 \mathrm{Mb}, 1-5 \mathrm{Mb}$ and $>5 \mathrm{Mb}$ and were identified as Small, Medium and Large, respectively. Three metrics were used to estimate levels of inbreeding: (1) $\mathrm{F}_{\mathrm{HOM}}$ was assessed based on the proportion of homozygotes using PLINK v1.07 software. (2) $\mathrm{F}_{\mathrm{ROH}}$ was calculated as described by McQuillan et al. (an individual's summed ROH length was normalized by the length of the autosomal genome covered by SNPs) ${ }^{67}$. (3) $\mathrm{F}_{\mathrm{GRM}}$ was estimated based on the variance of additive genotypes using GCTA 1.19 .2 method according to Yang et al. ${ }^{68}$. Correlations of inbreeding coefficient for three methods were estimated using cor.test function in R v3.2.4.

Statistical analysis of the genomic ROH across breeds. The correlations between the average proportion of the genome coverage by $\mathrm{ROH}$ and average observed heterozygosity (Ho) for each breed were estimated by cor.test functions in $\mathrm{R} v 3.4$.2. The $\mathrm{ROH}$ pattern based on the total number of $\mathrm{ROH}$ per individual and the total length of those $\mathrm{ROH}$ were measured for three classes. For analysis of distribution of ROH across genome, each chromosome was divided into 20 equal size segments, and their relative distribution over these chromosomal segments were calculated across breeds. All plots in current study were generated with the R programing (v.3.2.4).

Genomic regions within $\mathrm{ROH}$. To investigate genomic regions that were associated with occurrences of $\mathrm{ROH}$ within each breed, the fraction of SNPs in ROH was estimated based on the frequency of a SNP in them across individuals. 'ROH hotspot' was identified as a region of adjacent SNPs with an ROH frequency per SNP above the threshold of $1 \%$. Genomic coordinates for all identified $\mathrm{ROH}$ regions were used to annotate genes using the UMD3.1 genome assembly. The function of these genes and GO terms were further assessed using DAVID Functional Annotation Bioinformatics Microarray Analysis platform ${ }^{69,70}$.

Received: 11 October 2018; Accepted: 28 October 2019;

Published online: 18 November 2019 


\section{References}

1. Ceballos, F. C., Joshi, P. K., Clark, D. W., Ramsay, M. \& Wilson, J. F. Runs of homozygosity: windows into population history and trait architecture. Nature reviews. Genetics, https://doi.org/10.1038/nrg.2017.109 (2018).

2. Lander, E. S. \& Botstein, D. Homozygosity mapping: a way to map human recessive traits with the DNA of inbred children. Science 236, 1567-1570 (1987).

3. Rudan, I., Campbell, H., Carothers, A. D., Hastie, N. D. \& Wright, A. F. Contribution of consanguinuity to polygenic and multifactorial diseases. Nature genetics 38, 1224-1225, https://doi.org/10.1038/ng1106-1224 (2006).

4. Kirin, M. et al. Genomic runs of homozygosity record population history and consanguinity. PloS one 5, e13996, https://doi. org/10.1371/journal.pone.0013996 (2010).

5. Joshi, P. K. et al. Directional dominance on stature and cognition in diverse human populations. Nature 523, 459-462, https://doi. org/10.1038/nature14618 (2015).

6. Pemberton, T. J. et al. Genomic patterns of homozygosity in worldwide human populations. American journal of human genetics 91 , 275-292, https://doi.org/10.1016/j.ajhg.2012.06.014 (2012).

7. Nothnagel, M., Lu, T. T., Kayser, M. \& Krawczak, M. Genomic and geographic distribution of SNP-defined runs of homozygosity in Europeans. Human molecular genetics 19, 2927-2935, https://doi.org/10.1093/hmg/ddq198 (2010).

8. Peripolli, E. et al. Runs of homozygosity: current knowledge and applications in livestock. Animal genetics 48, 255-271, https://doi. org/10.1111/age.12526 (2017).

9. Mastrangelo, S. et al. Runs of homozygosity reveal genome-wide autozygosity in Italian sheep breeds. Animal genetics 49, 71-81, https://doi.org/10.1111/age.12634 (2018).

10. MacLeod, I. M., Larkin, D. M., Lewin, H. A., Hayes, B. J. \& Goddard, M. E. Inferring demography from runs of homozygosity in whole-genome sequence, with correction for sequence errors. Molecular biology and evolution 30, 2209-2223, https://doi. org/10.1093/molbev/mst125 (2013).

11. Purfield, D. C., Berry, D. P., McParland, S. \& Bradley, D. G. Runs of homozygosity and population history in cattle. BMC genetics 13, 70, https://doi.org/10.1186/1471-2156-13-70 (2012).

12. Bosse, M. et al. Regions of homozygosity in the porcine genome: consequence of demography and the recombination landscape. PLoS genetics 8, e1003100, https://doi.org/10.1371/journal.pgen.1003100 (2012).

13. Upadhyay, M. R. et al. Genetic origin, admixture and population history of aurochs (Bos primigenius) and primitive European cattle. Heredity 119, 469, https://doi.org/10.1038/hdy.2017.59 (2017).

14. Reverter, A. et al. Genomic inbreeding depression for climatic adaptation of tropical beef cattle. Journal of animal science $\mathbf{9 5}$, 3809-3821, https://doi.org/10.2527/jas2017.1643 (2017).

15. Bjelland, D. W., Weigel, K. A., Vukasinovic, N. \& Nkrumah, J. D. Evaluation of inbreeding depression in Holstein cattle using wholegenome SNP markers and alternative measures of genomic inbreeding. Journal of dairy science 96, 4697-4706, https://doi. org/10.3168/jds.2012-6435 (2013).

16. Martikainen, K., Tyriseva, A. M., Matilainen, K., Poso, J. \& Uimari, P. Estimation of inbreeding depression on female fertility in the Finnish Ayrshire population. Journal of animal breeding and genetics = Zeitschrift fur Tierzuchtung und Zuchtungsbiologie 134, 383-392, https://doi.org/10.1111/jbg.12285 (2017).

17. Peripolli, E. et al. Assessment of runs of homozygosity islands and estimates of genomic inbreeding in Gyr (Bos indicus) dairy cattle. BMC genomics 19, 34, https://doi.org/10.1186/s12864-017-4365-3 (2018).

18. Signer-Hasler, H. et al. Population structure and genomic inbreeding in nine Swiss dairy cattle populations. Genetics, selection, evolution: GSE 49, 83, https://doi.org/10.1186/s12711-017-0358-6 (2017).

19. Gomez-Raya, L., Rodriguez, C., Barragan, C. \& Silio, L. Genomic inbreeding coefficients based on the distribution of the length of runs of homozygosity in a closed line of Iberian pigs. Genetics, selection, evolution: GSE 47, 81, https://doi.org/10.1186/s12711-0150153-1 (2015).

20. Manunza, A. et al. A genome-wide perspective about the diversity and demographic history of seven Spanish goat breeds. Genetics, selection, evolution: GSE 48, 52, https://doi.org/10.1186/s12711-016-0229-6 (2016).

21. Upadhyay, M. R. et al. Genetic origin, admixture and population history of aurochs (Bos primigenius) and primitive European cattle. Heredity 118, 169-176, https://doi.org/10.1038/hdy.2016.79 (2017).

22. Zhang, Q., Guldbrandtsen, B., Bosse, M., Lund, M. S. \& Sahana, G. Runs of homozygosity and distribution of functional variants in the cattle genome. BMC genomics 16, 542, https://doi.org/10.1186/s12864-015-1715-x (2015).

23. Forutan, M. et al. Inbreeding and runs of homozygosity before and after genomic selection in North American Holstein cattle. BMC genomics 19, 98, https://doi.org/10.1186/s12864-018-4453-z (2018).

24. Kim, E. S., Sonstegard, T. S. \& Rothschild, M. F. Recent artificial selection in U.S. Jersey cattle impacts autozygosity levels of specific genomic regions. BMC genomics 16, 302, https://doi.org/10.1186/s12864-015-1500-x (2015).

25. Kim, E. S. et al. Effect of artificial selection on runs of homozygosity in u.s. Holstein cattle. PloS one 8, e80813, https://doi. org/10.1371/journal.pone.0080813 (2013).

26. Ferencakovic, M. et al. Estimates of autozygosity derived from runs of homozygosity: empirical evidence from selected cattle populations. Journal of animal breeding and genetics = Zeitschrift fur Tierzuchtung und Zuchtungsbiologie 130, 286-293, https://doi. org/10.1111/jbg.12012 (2013).

27. Metzger, J. et al. Runs of homozygosity reveal signatures of positive selection for reproduction traits in breed and non-breed horses. BMC genomics 16, 764, https://doi.org/10.1186/s12864-015-1977-3 (2015).

28. Mastrangelo, S. et al. Genome-wide scan for runs of homozygosity identifies potential candidate genes associated with local adaptation in Valle del Belice sheep. Genetics, selection, evolution: GSE 49, 84, https://doi.org/10.1186/s12711-017-0360-z (2017).

29. Szmatoła, T. et al. Characteristics of runs of homozygosity in selected cattle breeds maintained in Poland. Livestock Science 188, 72-80, https://doi.org/10.1016/j.livsci.2016.04.006 (2016).

30. Mei, C. et al. Genetic architecture and selection of Chinese cattle revealed by whole genome resequencing. Molecular biology and evolution, https://doi.org/10.1093/molbev/msx322 (2017).

31. Gao, Y. et al. Species composition and environmental adaptation of indigenous Chinese cattle. Scientific reports 7, 16196, https://doi. org/10.1038/s41598-017-16438-7 (2017).

32. Yang, L. et al. Genome-wide analysis reveals differential selection involved with copy number variation in diverse Chinese Cattle. Scientific reports 7, 14299, https://doi.org/10.1038/s41598-017-14768-0 (2017).

33. Zhang, W. et al. Genome-wide assessment of genetic diversity and population structure insights into admixture and introgression in Chinese indigenous cattle. BMC genetics 19, 114, https://doi.org/10.1186/s12863-018-0705-9 (2018).

34. Quinlan, A. R. \& Hall, I. M. BEDTools: a flexible suite of utilities for comparing genomic features. Bioinformatics 26, 841-842, https://doi.org/10.1093/bioinformatics/btq033 (2010).

35. Ferencakovic, M., Solkner, J. \& Curik, I. Estimating autozygosity from high-throughput information: effects of SNP density and genotyping errors. Genetics, selection, evolution: GSE 45, 42, https://doi.org/10.1186/1297-9686-45-42 (2013).

36. Marras, G. et al. Analysis of runs of homozygosity and their relationship with inbreeding in five cattle breeds farmed in Italy. Animal genetics 46, 110-121, https://doi.org/10.1111/age.12259 (2015).

37. Mastrangelo, S. et al. Genomic inbreeding estimation in small populations: evaluation of runs of homozygosity in three local dairy cattle breeds. Animal: an international journal of animal bioscience 10, 746-754, https://doi.org/10.1017/S1751731115002943 (2016). 
38. Pryce, J. E., Haile-Mariam, M., Goddard, M. E. \& Hayes, B. J. Identification of genomic regions associated with inbreeding depression in Holstein and Jersey dairy cattle. Genetics, selection, evolution: GSE 46, 71, https://oi.org/10.1186/s12711-014-0071-7 (2014).

39. Szpiech, Z. A. et al. Long runs of homozygosity are enriched for deleterious variation. American journal of human genetics $\mathbf{9 3}$, 90-102, https://doi.org/10.1016/j.ajhg.2013.05.003 (2013).

40. Porto-Neto, L. R. et al. Genomic divergence of zebu and taurine cattle identified through high-density SNP genotyping. BMC genomics 14, 876, https://doi.org/10.1186/1471-2164-14-876 (2013).

41. Randhawa, I. A., Khatkar, M. S., Thomson, P. C. \& Raadsma, H. W. A Meta-Assembly of Selection Signatures in Cattle. PloS one 11, e0153013, https://doi.org/10.1371/journal.pone.0153013 (2016).

42. Lee, T. et al. Genetic variants and signatures of selective sweep of Hanwoo population (Korean native cattle). BMB reports 46 , 346-351 (2013)

43. Xu, L. et al. Genomic signatures reveal new evidences for selection of important traits in domestic cattle. Molecular biology and evolution 32, 711-725, https://doi.org/10.1093/molbev/msu333 (2015).

44. Taye, M. et al. Exploring evidence of positive selection signatures in cattle breeds selected for different traits. Mammalian genome: official journal of the International Mammalian Genome Society 28, 528-541, https://doi.org/10.1007/s00335-017-9715-6 (2017).

45. Bahbahani, H. et al. Signatures of positive selection in East African Shorthorn Zebu: A genome-wide single nucleotide polymorphism analysis. Scientific reports 5, 11729, https://doi.org/10.1038/srep11729 (2015).

46. He, H., Chen, S., Liang, W. \& Liu, X. Genome-wide proteomics analysis on longissimus muscles in Qinchuan beef cattle. Animal genetics 48, 131-140, https://doi.org/10.1111/age.12508 (2017).

47. Picard, B. et al. Inverse relationships between biomarkers and beef tenderness according to contractile and metabolic properties of the muscle. Journal of agricultural and food chemistry 62, 9808-9818, https://doi.org/10.1021/jf501528s (2014).

48. Guillemin, N., Bonnet, M., Jurie, C. \& Picard, B. Functional analysis of beef tenderness. Journal of proteomics 75, 352-365, https:// doi.org/10.1016/j.jprot.2011.07.026 (2011).

49. Rodrigues, R. T. et al. Differences in Beef Quality between Angus (Bos taurus taurus) and Nellore (Bos taurus indicus) Cattle through a Proteomic and Phosphoproteomic Approach. PloS one 12, e0170294, https://doi.org/10.1371/journal.pone.0170294 (2017).

50. Lai, F. N. et al. Whole-genome scanning for the litter size trait associated genes and SNPs under selection in dairy goat (Capra hircus). Scientific reports 6, 38096, https://doi.org/10.1038/srep38096 (2016).

51. Guan, D. et al. Scanning of selection signature provides a glimpse into important economic traits in goats (Capra hircus). Scientific reports 6, 36372, https://doi.org/10.1038/srep36372 (2016).

52. Liu, Z. et al. Genome-wide analysis reveals signatures of selection for important traits in domestic sheep from different ecoregions. BMC genomics 17, 863, https://doi.org/10.1186/s12864-016-3212-2 (2016).

53. Perez, R., Canon, J. \& Dunner, S. Genes associated with long-chain omega-3 fatty acids in bovine skeletal muscle. Journal of applied genetics 51, 479-487, https://doi.org/10.1007/BF03208877 (2010).

54. Hiller, B., Angulo, J., Olivera, M., Nuernberg, G. \& Nuernberg, K. How selected tissues of lactating holstein cows respond to dietary polyunsaturated fatty acid supplementation. Lipids 48, 357-367, https://doi.org/10.1007/s11745-012-3737-3 (2013).

55. Hiller, B., Herdmann, A. \& Nuernberg, K. Dietary n-3 fatty acids significantly suppress lipogenesis in bovine muscle and adipose tissue: a functional genomics approach. Lipids 46, 557-567, https://doi.org/10.1007/s11745-011-3571-z (2011).

56. Rincon, G., Farber, E. A., Farber, C. R., Nkrumah, J. D. \& Medrano, J. F. Polymorphisms in the STAT6 gene and their association with carcass traits in feedlot cattle. Animal genetics 40, 878-882, https://doi.org/10.1111/j.1365-2052.2009.01934.x (2009).

57. Gui, L. S. et al. Genetic variants in the SIRT6 transcriptional regulatory region affect gene activity and carcass quality traits in indigenous Chinese beef cattle (Bos taurus). BMC genomics 19, 785, https://doi.org/10.1186/s12864-018-5149-0 (2018).

58. Poulsen, N. A., Robinson, R. C., Barile, D., Larsen, L. B. \& Buitenhuis, B. A genome-wide association study reveals specific transferases as candidate loci for bovine milk oligosaccharides synthesis. BMC genomics 20, 404, https://doi.org/10.1186/s12864019-5786-y (2019).

59. Ha, N. T. et al. Liver transcriptome analysis reveals important factors involved in the metabolic adaptation of the transition cow. Journal of dairy science 100, 9311-9323, https://doi.org/10.3168/jds.2016-12454 (2017).

60. Dunner, S. et al. Genes involved in muscle lipid composition in 15 European Bos taurus breeds. Animal genetics 44, 493-501, https:// doi.org/10.1111/age.12044 (2013)

61. Lee, S. H. et al. Use of a bovine genome array to identify new biological pathways for beef marbling in Hanwoo (Korean Cattle). BMC genomics 11, 623, https://doi.org/10.1186/1471-2164-11-623 (2010).

62. Seabury, C. M. et al. Genome-wide association study for feed efficiency and growth traits in U.S. beef cattle. BMC genomics 18, 386, https://doi.org/10.1186/s12864-017-3754-y (2017).

63. Totty, M. L., Morrell, B. C. \& Spicer, L. J. Fibroblast growth factor 9 (FGF9) regulation of cyclin D1 and cyclin-dependent kinase-4 in ovarian granulosa and theca cells of cattle. Molecular and cellular endocrinology 440, 25-33, https://doi.org/10.1016/j. mce.2016.11.002 (2017).

64. Beecher, C. et al. Polymorphisms in bovine immune genes and their associations with somatic cell count and milk production in dairy cattle. BMC genetics 11, 99, https://doi.org/10.1186/1471-2156-11-99 (2010).

65. Howrigan, D. P., Simonson, M. A. \& Keller, M. C. Detecting autozygosity through runs of homozygosity: a comparison of three autozygosity detection algorithms. BMC genomics 12, 460, https://doi.org/10.1186/1471-2164-12-460 (2011).

66. Purcell, S. et al. PLINK: a tool set for whole-genome association and population-based linkage analyses. American journal of human genetics 81, 559-575, https://doi.org/10.1086/519795 (2007).

67. McQuillan, R. et al. Runs of homozygosity in European populations. American journal of human genetics 83, 359-372, https://doi. org/10.1016/j.ajhg.2008.08.007 (2008).

68. Yang, J., Lee, S. H., Goddard, M. E. \& Visscher, P. M. GCTA: a tool for genome-wide complex trait analysis. American journal of human genetics 88, 76-82, https://doi.org/10.1016/j.ajhg.2010.11.011 (2011).

69. Huang da, W., Sherman, B. T. \& Lempicki, R. A. Systematic and integrative analysis of large gene lists using DAVID bioinformatics resources. Nature protocols 4, 44-57, https://doi.org/10.1038/nprot.2008.211 (2009).

70. Huang da, W., Sherman, B. T. \& Lempicki, R. A. Bioinformatics enrichment tools: paths toward the comprehensive functional analysis of large gene lists. Nucleic acids research 37, 1-13, https://doi.org/10.1093/nar/gkn923 (2009).

\section{Acknowledgements}

This study was supported by the Basic R\&D Fund for the Central Level Scientific Research Institute (2016ywf-yb-6), National Beef Cattle Industrial Technology System (CARS-37), the Agricultural Science and Technology Innovation Program in Chinese Academy of Agricultural Sciences (ASTIP-IAS-TS-16 and ASTIPIAS03). L.Y.X was supported by the Elite Youth Program in Chinese Academy of Agricultural Sciences. The project was also partly supported by Beijing City Board of Education Foundation (PXM2016_014207_000012) for the data analysis and interpretation of the study. 


\section{Author contributions}

Conceived and designed the experiments: L.Y.X., J.Y.L. and G.E.L. Analyzed the data: G.Y.Z., L.Y., H.J.G., B.Z. and L.Y.X. Contributed reagents/materials/analysis tools: L.P.Z., Y.C. and X.G. Wrote the paper: L.Y.X., G.E.L. and J.Y.L. All authors read and approved the final manuscript.

\section{Competing interests}

The authors declare no competing interests.

\section{Additional information}

Supplementary information is available for this paper at https://doi.org/10.1038/s41598-019-53274-3.

Correspondence and requests for materials should be addressed to L.X. or J.L.

Reprints and permissions information is available at www.nature.com/reprints.

Publisher's note Springer Nature remains neutral with regard to jurisdictional claims in published maps and institutional affiliations.

(c) (i) Open Access This article is licensed under a Creative Commons Attribution 4.0 International License, which permits use, sharing, adaptation, distribution and reproduction in any medium or format, as long as you give appropriate credit to the original author(s) and the source, provide a link to the Creative Commons license, and indicate if changes were made. The images or other third party material in this article are included in the article's Creative Commons license, unless indicated otherwise in a credit line to the material. If material is not included in the article's Creative Commons license and your intended use is not permitted by statutory regulation or exceeds the permitted use, you will need to obtain permission directly from the copyright holder. To view a copy of this license, visit http://creativecommons.org/licenses/by/4.0/.

(c) The Author(s) 2019 